\title{
Shen-fu injection alleviates acute renal injury by reducing cytokine levels and modulating apoptosis in a porcine hemorrhagic shock model
}

\author{
Wei Yuan ${ }^{1}\left(\mathbb{D}\right.$, JunYuan $W^{1}{ }^{(D)}$, Qiang Zhang ${ }^{2}$, Yong Liang $^{1} \mathbb{D}^{D}$, MingQqing Zhang ${ }^{3}$ (D) HongJie Qin ${ }^{4}$ \\ Chun-Sheng $\mathrm{Li}^{1, *}$ \\ 1.PhD. Capital Medical University - Beijing ChaoYang Hospital - Department of Emergency - Beijing, China. \\ 2.PhD. Peking University Third Hospital - Department of Critical Care Medicine - Beijing, China. \\ 3.Master. Beijing Jishuitan Hospital - Department of Emergency Medicine - Beijing, China. \\ 4. Master. Capital Medical University - Beijing Luhe Hospital - Department of Emergency Medicine - Beijing, China.
}

\begin{abstract}
Purpose: Shen-fu injection (SFI) was used to intervene in the resuscitation of porcine hemorrhagic shock (HS) model to study its protective effects on acute kidney injury. Methods: After 60 min of HS, 28 animals were randomly assigned into four groups. The groups were as follows: hemorrhagic shock group (HS); HS resuscitation with shed-blood group (HSR); HS resuscitation with shed-blood and SFI ( $\left.1 \mathrm{~mL} \cdot \mathrm{kg}^{-1}\right)$ group (HSR-SFI); and the sham operation group (Sham). The bloods were analyzed for serum creatinine ( $\mathrm{sCr}$ ), cystatin C (CysC) and neutrophil gelatinase-associated lipocalin (NGAL). BAX, Bcl-2, and caspase-3 protein expressions by Western blot analysis and immunohistochemical staining. The renal tissues were removed and pathologic changes were observed. Results: Mean aortic pressure (MAP) in HSR-SFI groups were higher than that in HSR groups after shock. At the 6th hour after shock, the urine volume per hour in the HSRSFI groups was more than that in the HSR groups. The SCr, NGAL, CysC and cytokine levels of HSR-SFI groups were lower. The Bcl-2 expression was increased in the HSR-SFI groups. The BAX and caspase-3 expressions were reduced. The histopathologic score in the HSR-SFI was lower. Conclusion: SFI may reduce the risk of acute kidney injury (AKI) following hemorrhagic shock by attenuating systemic inflammatory responses, and regulating the expression of apoptosis-related proteins.
\end{abstract}

Key words: Shock Hemorrhagic. Acute Kidney Injury. Cytokines. Apoptosis.

*Corresponding author: 2000ywei@sina.com

Received: Dec 18, 2020 | Review: Feb 21, 2021 | Accepted: Mar 16, 2021

Conflict of interest: Nothing to declare.

Research performed at the Department of Emergency, Beijing ChaoYang Hospital, Capital Medical University, Beijing, China. 


\section{Introduction}

Trauma is the third leading cause of death worldwide, and the leading cause of mortality in people older than 44 years $^{1}$. Secondary organ dysfunction is one of the main causes of trauma deaths. Acute kidney injury (AKI) is the most common complication after trauma with a reported incidence of $50 \%{ }^{2}$. Acute kidney injury may be caused by several reasons. Except for decreased renal perfusion that results from hemorrhage, rhabdomyolysis, and systematic inflammation, renal oxidative stress during resuscitation is the critical indicator associated with later $\mathrm{AKI}^{3-5}$. Reducing the incidence of AKI may reduce mortality of trauma patients.

Shen-fu injection (SFI) is traditionally Chinese, its main active ingredients are ginsenosides and aconitine alkaloids and its solvent is $100 \mathrm{~mL} 5 \%$ glucose injection ${ }^{6}$. The ginsenoside scavenges free radicals, inhibiting inflammatory mediators ${ }^{7,8}$, suppressing cellular apoptosis ${ }^{9}$. This traditional Chinese medicine has been routinely administered to treat septic shock, acute myocardial dysfunction, and chronic congestive heart failure and to boost postoperative recovery ${ }^{10,11}$. On this basis, the hypothesis is that SFI could protect renal function from circulatory insufficiency induced hemorrhage. Reports on the effect of ginseng on hemorrhagic rat led to the design of this study ${ }^{12}$, and this is the first comparative study of SFI in hemorrhagic shock (HS) porcine model. In this study, SFI was used to intervene in the resuscitation of porcine HS model to study its protective effects on AKI and to explore the mechanism of action.

\section{Methods}

The study experimental protocol was approved by the Committee on the Ethics of Animal Experiments of Capital Medical University (Permit No. 2010-D-013). The animal experiments were in compliance with the Guiding Principles for the Care and Use of Animals expressed in the Declaration of Helsinki ${ }^{13}$.

\section{Study subject characteristics}

Twenty-eight male Beijing Landrace pigs were used for this experiment. The pigs were $12 \pm 2$ months of age, weighed $28 \pm 2 \mathrm{~kg}$, and were provided by a registered laboratory animal center in Beijing, China. The animals were fasted overnight with free access to water before surgery.

\section{Anesthesia and perioperative management}

Initial sedation was induced by intramuscular injection of ketamine $\left(0.5 \mathrm{mg} \cdot \mathrm{kg}^{-1}\right)$, followed by intravenous injection of propofol $\left(1 \mathrm{mg} \cdot \mathrm{kg}^{-1}\right)$. Then, propofol $\left(9 \mathrm{mg} \cdot \mathrm{kg}^{-1} \cdot \mathrm{h}^{-1}\right)$ and fentanyl $\left(1 \mu \mathrm{g} \cdot \mathrm{kg}^{-1} \cdot \mathrm{h}^{-1}\right)$ were administered intravenously to maintain the anesthesia and analgesia. A cuffed 6.5- $\mathrm{mm}$ endotracheal tube was advanced into the trachea.

The pigs were mechanically ventilated with a volumecontrolled ventilator (Servo 900c; Siemens, Berlin, Germany) with a tidal volume of $8 \mathrm{~mL} \cdot \mathrm{kg}^{-1}$, a constant fraction of inspired oxygen of 0.21 , and an inspiration/expiration ratio of $1: 2$ with a positive end-expiratory pressure of $5 \mathrm{~cm} \mathrm{H}_{2} \mathrm{O}$. End-tidal $\mathrm{PaCO} 2$ was monitored with an in-line infrared capnograph system ( $\mathrm{CO}_{2} \mathrm{SMO}$ Plus monitor; Respironics, Inc, Murrysville, PA, USA). Respiratory frequency was adjusted to maintain end-tidal $\mathrm{PaCO} 2$ between 35 and $40 \mathrm{mmHg}$.

The right femoral artery and right external jugular vein were exposed. A 6F catheter (Edwards Lifesciences, Irvine, CA) was advanced from the right femoral artery into the thoracic aorta to collect arterial blood samples and to measure the mean aortic pressure (MAP) using a pressure transducer (Biosensors International Group, Singapore).

The arterial and central venous catheters were connected to an integrated bedside monitor (PiCCO; Pulsion Medical Systems, Munich, Germany) for continuous hemodynamic monitoring including measure right atrial pressure and cardiac output (CO). All catheters were calibrated before use, and tip positions were confirmed by the presence of pressure traces. The electrocardiograph and all hemodynamic parameters were monitored using a multifunction monitor (M1165; Hewlett Packard Enterprise, Palo Alto, CA).

\section{Experimental protocol}

After surgery, the pigs achieved a stable resting level, and baseline data were recorded. The animal model of HS was adapted according to the previous study ${ }^{14}$. Pigs were rapidly bled via arterial sheath in the inguinal to a mean arterial pressure of $40 \mathrm{mmHg}$ within $10 \mathrm{~min}$ and were maintained at $40 \pm 3 \mathrm{mmHg}$ for $60 \mathrm{~min}$.

Blood was stored in a blood preservation bag (S-400, Sichuang Nightingale Biological Co. Ltd). Additional blood was withdrawn at a mean arterial pressure of $44 \mathrm{mmHg}$, and normal saline was infused at a mean arterial pressure of $36 \mathrm{mmHg}$.

After 60 min of HS, 28 animals were randomly assigned into four groups by resuscitation method. The groups were as follows: HS without resuscitation (HS), HS resuscitation with shed-blood group (HSR), HS resuscitation with shedblood and SFI ( $\left.1 \mathrm{~mL} \cdot \mathrm{kg}^{-1}\right)$ group (HSR-SFI), with eight animals in each of the three groups; and the sham operation group (Sham), which had four animals. Shen-fu injection $\left(1 \mathrm{~mL} \cdot \mathrm{kg}^{-1}\right)$ was administered in a single dose chosen based on the findings of previous experiments ${ }^{15}$. 
Further, the pigs were administered a basal normal saline infusion of $10 \mathrm{~mL} \cdot \mathrm{kg}^{-1} \cdot \mathrm{h}^{-1}$. They were euthanized with intravenous propofol $\left(3 \mathrm{mg} \cdot \mathrm{kg}^{-1}\right)$, followed by potassium chloride $\left(10 \mathrm{~mL}\right.$ of $\left.10 \mathrm{~mol} \cdot \mathrm{L}^{-1}\right) 6 \mathrm{~h}$ after $\mathrm{HS}$.

\section{Outcome measurements}

\section{Hemodynamic parameters}

Hemodynamic parameters were continuously monitored and regularly recorded. Heart rate (HR), MAP and intrathoracic blood volume index (ITBVI) were measured at baseline, and at 1,4 and $6 \mathrm{~h}$ post shock. Urine collected via a Foley catheter, and the urine output volumes were recorded per hour.

\section{Blood samples}

Venous blood was collected at baseline and at 1, 4 and $6 \mathrm{~h}$ following HS. The blood samples were used to measure serum creatinine $(\mathrm{sCr})$, neutrophil gelatinaseassociated lipocalin (NGAL), and cystatin C (CysC) levels, and all biomarkers were measured in duplicate by a single enzyme-linked immunosorbent assay (ELISA). Cytokine levels in plasma were analyzed at baseline and at 1,4 , and $6 \mathrm{~h}$ after HS. Tumor necrosis factor $\alpha$ (TNF- $\alpha$ ), interleukin-1 beta (IL-1 $\beta$ ), and interleukin- 6 (IL-6) levels were determined by a quantitative sandwich ELISA using commercially available kits (RayBiotech, USA) specific for porcine cytokines.

\section{Western blot}

Part of the renal cortex was fixed in $4 \%$ paraformaldehyde, embedded in paraffin, and sliced to produce three sections per sample. BCL2-Associated X (BAX), B-cell lymphoma-2 $(\mathrm{Bcl}-2)$ and caspase-3 expression were measured using the streptavidin peroxidase method with immunohistochemistry kits (Santa Cruz Co., USA).

Five high-power fields were chosen and examined by light microscopy. The BAX, Bcl-2 and caspase- 3 protein expressions in each section was represented by the integral optical density (IOD) and was analyzed using Image Pro Plus 6.0 software (Media Cybernetics, Inc., USA).

\section{Immunohistochemical staining}

Immunohistochemical staining was performed on the fixed kidney tissue slides using a standard protocol with primary antibodies, including monoclonal anti-BAX (50599-2-lg, Proteintech), anti-Bcl-2 antibodies (12789-1-AP, Proteintech), and anti-caspase-3 (19677-1-AP, Proteintech) at 1:10,000 dilution (Cell Signaling Technology; Denver, USA) and secondary horseradish peroxidase-conjugated goat anti-mouse antibody at 1:1,000 dilution. The staining results were observed under optical microscopy (CX41; Olympus, Tokyo, Japan).

The IOD values of tissue sections in each group were measured by Image-Pro Plus 6.0 software (Media Cybernetics, Inc., Bethesda, MD, USA) after tissue images were captured under an optical microscope (400x). Five views were randomly selected to determine the positive IOD values, and the mean IOD values were defined as the relative expressions of $\mathrm{BAX}, \mathrm{Bcl}-2$, and caspase-3.

\section{Renal tissue sampling}

The kidney was surgically removed and preserved in $10 \%$ formaldehyde or $4 \%$ paraformaldehyde to observe pathologic changes under a light microscope. Kidney damage was scored by grading any glomerular, tubular, and interstitial change, based on a previous study ${ }^{16}$. The sum of the partial scores resulted in a final grade from zero to nine. Another portion of the kidney tissue was preserved to observe changes in ultra-microstructure under a transmission electron microscope. The pathologic evaluations were performed by an independent pathologist who has more than ten years of experience and was blinded to this study.

\section{Statistical analysis}

Statistical analyses were performed with SPSS 19.0 software (SPSS, Chicago, IL, USA). Values are shown as mean \pm standard deviation. Continuous variables were compared between groups. Repeated measures analysis of variance (ANOVA) was used to determine differences over time within groups, as appropriate. Least significant difference (LSD) method was used for paired groups comparisons. A two-sided $p$-value $<0.05$ was considered statistically significant.

\section{Results}

\section{Hemodynamic parameters}

In the three experimental groups, the HRs were higher than that of the sham group one hour after reaching the target blood pressure of $40 \mathrm{mmHg}(p<0.001)$. There was no significant difference in HR between the HSR and HSR-SFI groups during resuscitation, either at 4 or $6 \mathrm{~h}$. Mean aortic pressure in the HSR-SFI group was higher than that in the HSR group at 4 and $6 \mathrm{~h}$ post shock $\left(p_{4 h}=0.040\right.$, $\left.p_{6 h}=0.048\right)$. No significant differences in ITBVI between the HSR and HRS-SFI groups during the experimental protocol were found (Table 1). 
Table 1 - Comparison of hemodynamic indexes and renal function in each group.

\begin{tabular}{|c|c|c|c|c|}
\hline \multirow{2}{*}{ Group } & \multicolumn{4}{|c|}{ Period } \\
\hline & Baseline & 1 hour & 4 hours & 6 hours \\
\hline \multicolumn{5}{|l|}{ HR (bpm) } \\
\hline Sham $(n=4)$ & $116.25 \pm 16.17$ & $117.25 \pm 3.30$ & $115.50 \pm 9.33$ & $106.75 \pm 10.97$ \\
\hline $\mathrm{HS}(\mathrm{n}=8)$ & $127.63 \pm 25.35$ & $200.25 \pm 16.54^{b}$ & $224.75 \pm 9.82^{b}$ & $222.75 \pm 12.96^{b}$ \\
\hline $\operatorname{HSR}(n=8)$ & $126.00 \pm 25.84$ & $213.25 \pm 29.54$ & $181.13 \pm 19.87$ & $184.75 \pm 20.15$ \\
\hline HSR-SFI $(n=8)$ & $118.38 \pm 30.91$ & $216.63 \pm 27.81$ & $191.63 \pm 14.89$ & $189.38 \pm 34.13$ \\
\hline \multicolumn{5}{|l|}{ MAP (mmHg) } \\
\hline Sham & $111.50 \pm 13.77$ & $111.50 \pm 7.14$ & $109.25 \pm 7.09$ & $103.50 \pm 11.73$ \\
\hline HS & $110.88 \pm 9.82$ & $40.13 \pm 2.03^{b}$ & $69.13 \pm 9.73^{b}$ & $55.13 \pm 9.96^{b}$ \\
\hline HSR & $112.63 \pm 14.70$ & $39.75 \pm 1.67$ & $94.88 \pm 8.66$ & $105.13 \pm 7.20$ \\
\hline HSR-SFI & $102.63 \pm 19.66$ & $40.13 \pm 2.03$ & $104.75 \pm 9.54^{c}$ & $114.50 \pm 9.74^{c}$ \\
\hline \multicolumn{5}{|l|}{ ITBVI $\left(\mathbf{m L} \cdot \mathbf{m}^{-2}\right)$} \\
\hline Sham & $604.00 \pm 120.72$ & $572.25 \pm 33.09$ & $593.00 \pm 73.23$ & $497.00 \pm 72.26$ \\
\hline HS & $606.75 \pm 136.92$ & $322.75 \pm 93.88^{b}$ & $345.63 \pm 110.15^{a}$ & $347.00 \pm 108.54^{a}$ \\
\hline HSR & $599.75 \pm 150.08$ & $383.75 \pm 73.61$ & $708.25 \pm 152.94$ & $621.25 \pm 67.86$ \\
\hline HSR-SFI & $598.88 \pm 115.78$ & $430.88 \pm 120.01$ & $634.13 \pm 128.05$ & $573.88 \pm 51.94$ \\
\hline \multicolumn{5}{|c|}{ UV/per $\left(\mathrm{mL} \cdot\right.$ hour $\left.^{-1}\right)$} \\
\hline Sham & $94.38 \pm 10.43$ & $95.88 \pm 8.11$ & $103.00 \pm 8.08$ & $97.75 \pm 8.73$ \\
\hline HS & $86.41 \pm 13.15$ & $9.50 \pm 4.34^{b}$ & $8.88 \pm 6.42^{b}$ & $6.88 \pm 7.74^{b}$ \\
\hline HSR & $91.16 \pm 8.19$ & $13.88 \pm 7.00$ & $64.38 \pm 11.44$ & $77.50 \pm 9.49$ \\
\hline HSR-SFI & $92.66 \pm 6.82$ & $13.00 \pm 4.72$ & $61.13 \pm 11.41$ & $92.63 \pm 11.65^{c}$ \\
\hline \multicolumn{5}{|l|}{$\operatorname{sCr}\left(\right.$ umol. $\left.\mathrm{L}^{-1}\right)$} \\
\hline Sham & $101.03 \pm 4.44$ & $96.15 \pm 8.72$ & $98.73 \pm 10.28$ & $92.20 \pm 8.55$ \\
\hline HS & $96.13 \pm 7.73$ & $119.30 \pm 12.49^{a}$ & $178.04 \pm 30.55^{a}$ & $358.20 \pm 59.64^{b}$ \\
\hline HSR & $98.40 \pm 6.78$ & $121.81 \pm 14.41^{\mathrm{a}}$ & $173.96 \pm 38.11$ & $220.69 \pm 54.97$ \\
\hline HSR-SFI & $99.69 \pm 4.75$ & $127.83 \pm 23.80^{\mathrm{a}}$ & $169.20 \pm 45.23$ & $169.20 \pm 45.23^{c}$ \\
\hline \multicolumn{5}{|l|}{ NGAL (ng. mL $\left.{ }^{-1}\right)$} \\
\hline Sham & $1.49 \pm 0.40$ & $1.34 \pm 0.27$ & $1.45 \pm 0.36$ & $1.38 \pm 0.36$ \\
\hline HS & $1.46 \pm 0.43$ & $1.50 \pm 0.33$ & $5.08 \pm 0.96^{d}$ & $9.12 \pm 0.90^{d}$ \\
\hline HSR & $1.54 \pm 0.56$ & $1.77 \pm 0.40$ & $4.85 \pm 0.89$ & $7.43 \pm 1.46$ \\
\hline HSR-SFI & $1.57 \pm 0.51$ & $1.93 \pm 0.51$ & $4.49 \pm 0.69$ & $5.40 \pm 1.35^{c}$ \\
\hline \multicolumn{5}{|l|}{ CysC (ug. $\left.L^{-1}\right)$} \\
\hline Sham & $535.75 \pm 44.78$ & $507.30 \pm 59.80$ & $498.75 \pm 22.27$ & $527.28 \pm 41.77$ \\
\hline HS & $544.10 \pm 39.91$ & $531.59 \pm 34.21$ & $665.34 \pm 66.79^{b}$ & $749.58 \pm 67.47^{b}$ \\
\hline HSR & $532.59 \pm 46.44$ & $564.75 \pm 45.64$ & $593.62 \pm 47.78$ & $664.90 \pm 68.23$ \\
\hline HSR-SFI & $524.20 \pm 29.39$ & $539.11 \pm 27.28$ & $573.44 \pm 28.95$ & $577.30 \pm 37.17^{c}$ \\
\hline
\end{tabular}

Values are mean \pm SE. ap $<0.05$ vs. sham; bp $<0.001$ vs. sham; $c p<0.05$ vs. HSR; $d p<0.001$ vs. HSR. Sham: the sham operation group, HS: the hemorrhagic shock group, HSR: the hemorrhagic shock and resuscitation group, HSR-SFI: the hemorrhagic shock and Shen-fu injection resuscitation group. HR: heart rate, MAP: mean arterial pressure, ITBVI: intrathoracic blood volume index, UV: urine volume, sCr: serum creatinine, NGAL: neutrophil gelatinase associated lipocalin, CysC: cystatin C.

\section{Renal function and biomarkers of AKI}

One hour post shock, the urine volume per hour in the three experimental groups was significantly less than that in the sham group $(p<0.001)$. Six hours post-shock, the urine output per hour in the HSR-SFI group was higher than in the HSR group $(p=0.004)$. Additionally, at $6 \mathrm{~h}$ post shock, the sCr level in the HSR-SFI group was lower than that in the $\operatorname{HSR}$ group $(p=0.037)$ (Table 1$)$.

Levels of NGAL and CysC in the HS group were higher than those in the sham group at $1 \mathrm{~h}$ post shock. In the HSR-SFI groups, the biomarkers were lower in the two resuscitation groups at $6 \mathrm{~h}$ post shock after (NGAL: $\left.\mathrm{p}_{6 \mathrm{~h}}=0.002 ; \mathrm{CysC}: \mathrm{p}_{6 \mathrm{~h}}=0.006\right)$. 


\section{Plasma cytokine levels}

At the 4 and $6 \mathrm{~h}$ intervals post shock, cytokine levels for TNF- $\alpha$, IL-1 $\beta$, and IL- 6 in the HS group were significantly higher than those in the sham group $(p<0.001)$. Six hours post shock, the cytokine levels in the HSR-SFI groups were lower than those in HSR groups $\left(\mathrm{p}_{\mathrm{TNF}-\alpha}=0.009 ; \mathrm{p}_{\mathrm{IL}-1 \beta}=0.005\right.$; $\mathrm{p}_{\mathrm{IL}-6}=0.001$ ) (Fig. 1).

\section{Protein expression in the apoptotic pathway}

After Western blot analysis, compared to the sham group, the expressions of $\mathrm{BCl}-2$ and $\mathrm{BAX}$ were significantly increased in the HS groups $(p<0.001)$. Correspondingly, caspase- 3 expression in the HS groups was higher than that in the sham groups $(p<0.001)$. Between the two resuscitation groups, $\mathrm{BCl}-2$ expression was significantly increased $(\mathrm{p}<0.001)$ and BAX expression was slightly reduced $(p=0.038)$ in the HSR-SFI groups. caspase-3 expression in the HSR-SFI groups was lower than that in the HSR groups $(p=0.012)$ (Fig. 2).

\section{Immunohistochemical staining}

The positive expression of an apoptosis-related protein in each group was observed. The expression of $\mathrm{Bcl}-2$

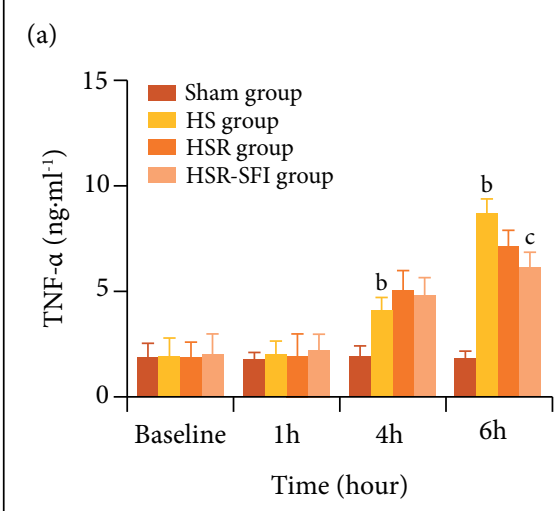

(b)

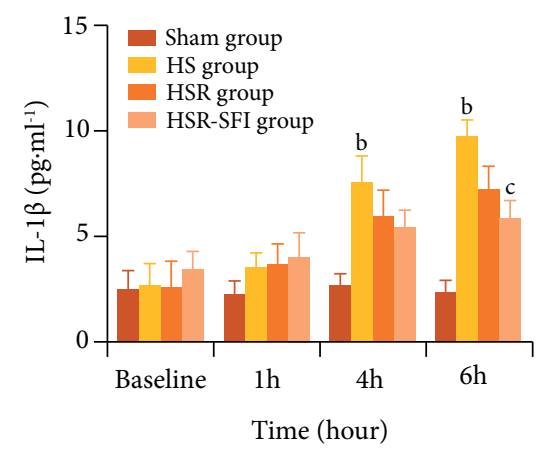

(c)

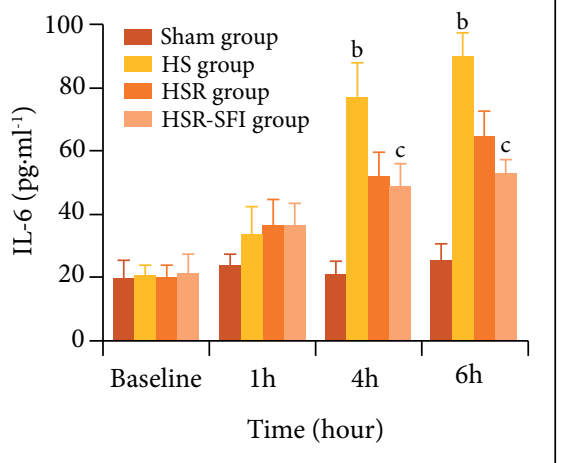

Figure 1 - The plasma cytokine levels in four groups: $a, p<0.05$ vs. sham; $b, p<0.001$ vs. sham; $c, p<0.05$ vs. HSR; $d, p<0.001$ vs. HSR. (a) Tumor necrosis factor $\alpha$; (b) Interleukin-1 beta; (c) Interleukin-6. At 4 and $6 \mathrm{~h}$ after shock, all cytokine levels (TNF- $\alpha$, IL-1 $\beta$ and IL-6) in HS group were significantly higher than those in Sham group ( $p<0.001)$. And at $6 \mathrm{~h}$ after shock, the cytokine levels in HSR-SFI groups were lower than those in HSR groups (pTNF- $\alpha=0.0170 .009$; plL-1 $\beta=0.0160 .005 ;$ plL- $6=0.0030 .001$ ). Sham: the sham operation group, HS: the hemorrhagic shock group, HSR: the hemorrhagic shock and resuscitation group, HSR-SFI: the hemorrhagic shock and Shen-fu injection resuscitation group.

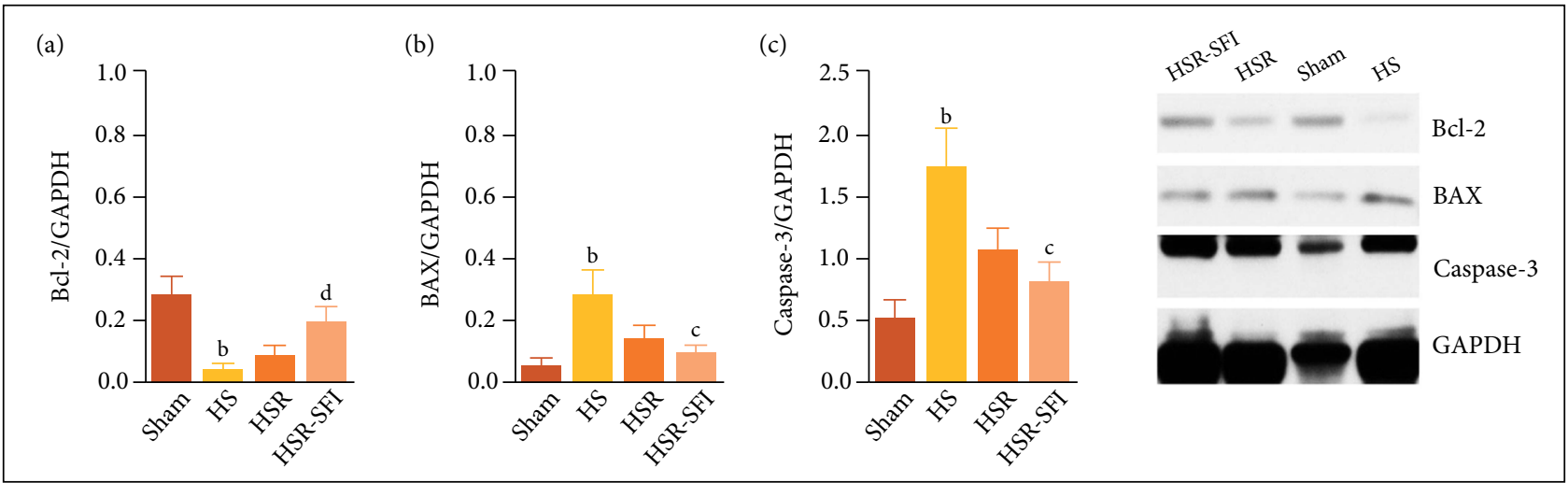

Figure 2 - The expression of (a) Bcl-2, (b) BAX and (c) caspase-3 proteins. a,p $<0.05$ vs. sham; b,p $<0.001$ vs. sham; c, $p<0.05$ vs. HSR; $d, p<0.001$ vs. HSR compared with the Sham groups, Bcl-2 expression was significantly decreased, and BAX expression was increased in the HS groups $(p<0.001)$. Correspondingly, caspase-3 expression in the HS groups was higher than that in the sham groups $(\mathrm{p}<0.001)$. Between the two resuscitation groups, $\mathrm{Bcl}-2$ expression was significantly increased in the HSR-SFI groups $(p<0.001)$, and BAX expression was slightly reduced ( $p=0.038)$. Caspase-3 expression in the HSR-SFI groups was lower than that in the HSR groups $(p=0.012)$. Sham: the sham operation group, HS: the hemorrhagic shock group, HSR: the hemorrhagic shock and resuscitation group, HSR-SFI: the hemorrhagic shock and Shen-fu injection resuscitation group. 
protein was lower $(p=0.032)$ and that of BAX was higher $(p=0.003)$ in the HS group than in the sham group. The expression of caspase- 3 in the HS group was higher than that in the sham group $(p=0.034)$. In the two resuscitation groups, the expression of $\mathrm{Bcl}-2$ in the HSR-SFI group was higher $(\mathrm{p}=0.031)$ and that of $B A X$ and caspase-3 were lower $\left(p_{B A X}=0.041\right.$, $\left.\mathrm{p}_{\text {caspase-3 }}=0.026\right)($ Fig. 3).

\section{Renal histopathology}

In the HS groups, the results of light microscopy showed that the glomerular capillaries are closed, the whole glomerulus becomes smaller, and the endothelial cells of the glomerulus and the outer poetasters are in a state of pyknosis (Fig. 4, indicated by a black arrow). The renal microcystitis was widened, and there were some fine particles in the cavity. The epithelium of the proximal convoluted tubules is slightly lower than that of the normal ones; the intercellular boundary is not clear. A fine granular substance is scattered in the lumen.

Electron microscopy revealed that in the HS groups the microvilli on the free surface of the proximal convoluted tubules were incomplete, the mitochondria in the epithelial cells were significantly expanded, the arrangement of mitochondria cristae was disordered, and even disappeared (indicated by a white arrow). Owing to the expansion and deformation of mitochondria, the basal folds around them were disordered, and the lysosomes in the cells were increased. Autophagy was observed, and the deformed mitochondria were engulfed.

The histopathologic score in the HS group was significantly higher than that in the sham group $(p<0.001)$. Of the two resuscitation groups, the HSRSFI group had a lower histopathologic score $(p=0.004)$.

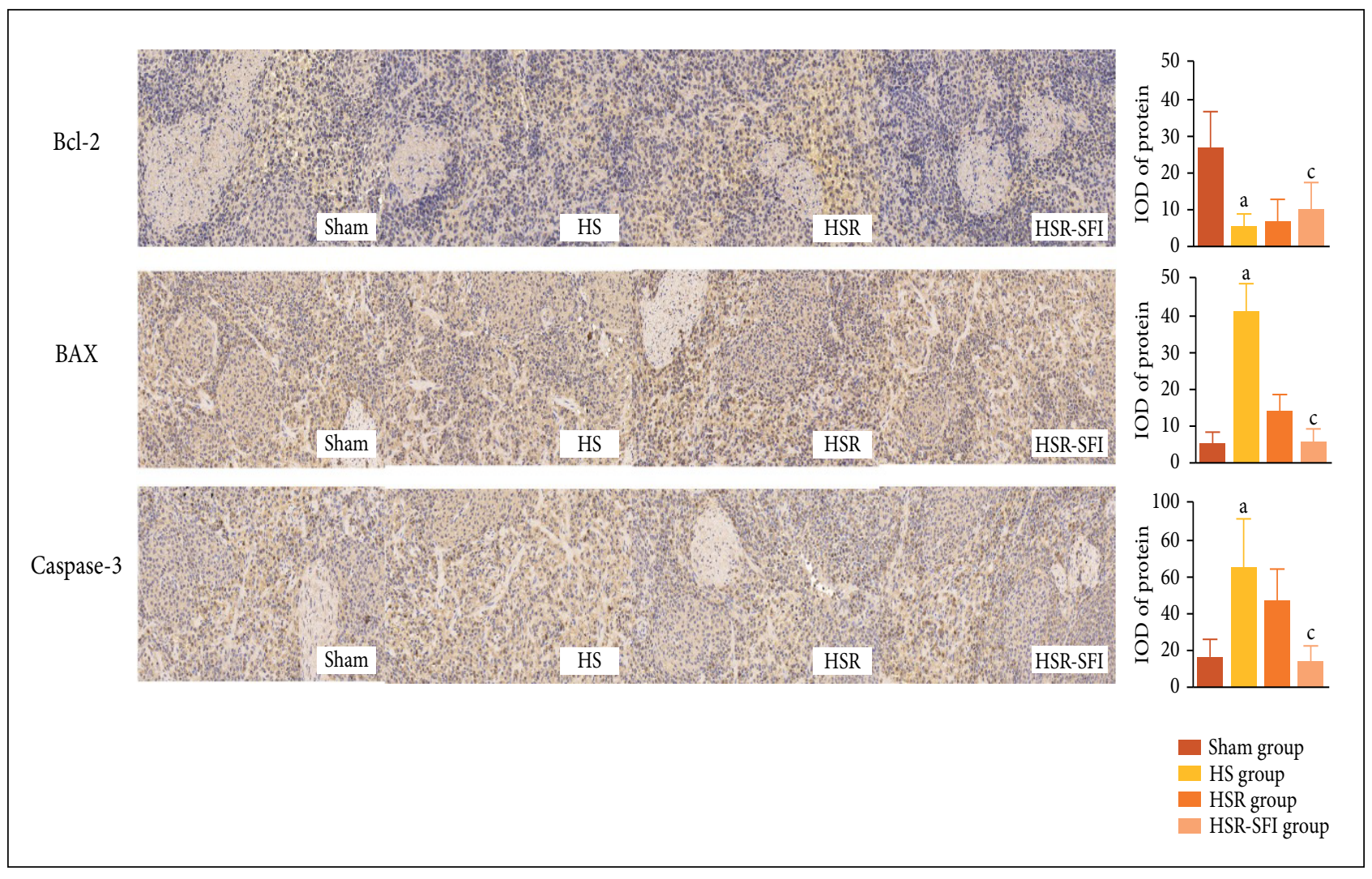

Figure 3 - The expression of Bcl-2, BAX and caspase-3 proteins in four groups. a,p $<0.05$ vs. sham; b, $p<0.001$ vs. sham; $c, p<0.05$ vs. HSR; $d, p<0.001$ vs. HSR. Compared with sham group, the positive expression of Bcl-2 protein was lower $(p=0.032)$ and BAX protein was higher $(p=0.003)$ in HS group. Accordingly, the positive expression of caspase-3 protein in HS group was higher than that in sham group $(p=0.034)$. In the two resuscitation groups, the positive expression of $\mathrm{Bcl}-2$ in HSR-SFI group was higher $(p=0.031)$, and the positive expression of BAX and caspase-3 proteins were lower $\left(p_{B A X}=0.041, p_{\text {caspase-3 }}=0.026\right)$. Sham: the sham operation group, HS: the hemorrhagic shock group, HSR: the hemorrhagic shock and resuscitation group, HSR-SFI: the hemorrhagic shock and Shen-fu injection resuscitation group. 


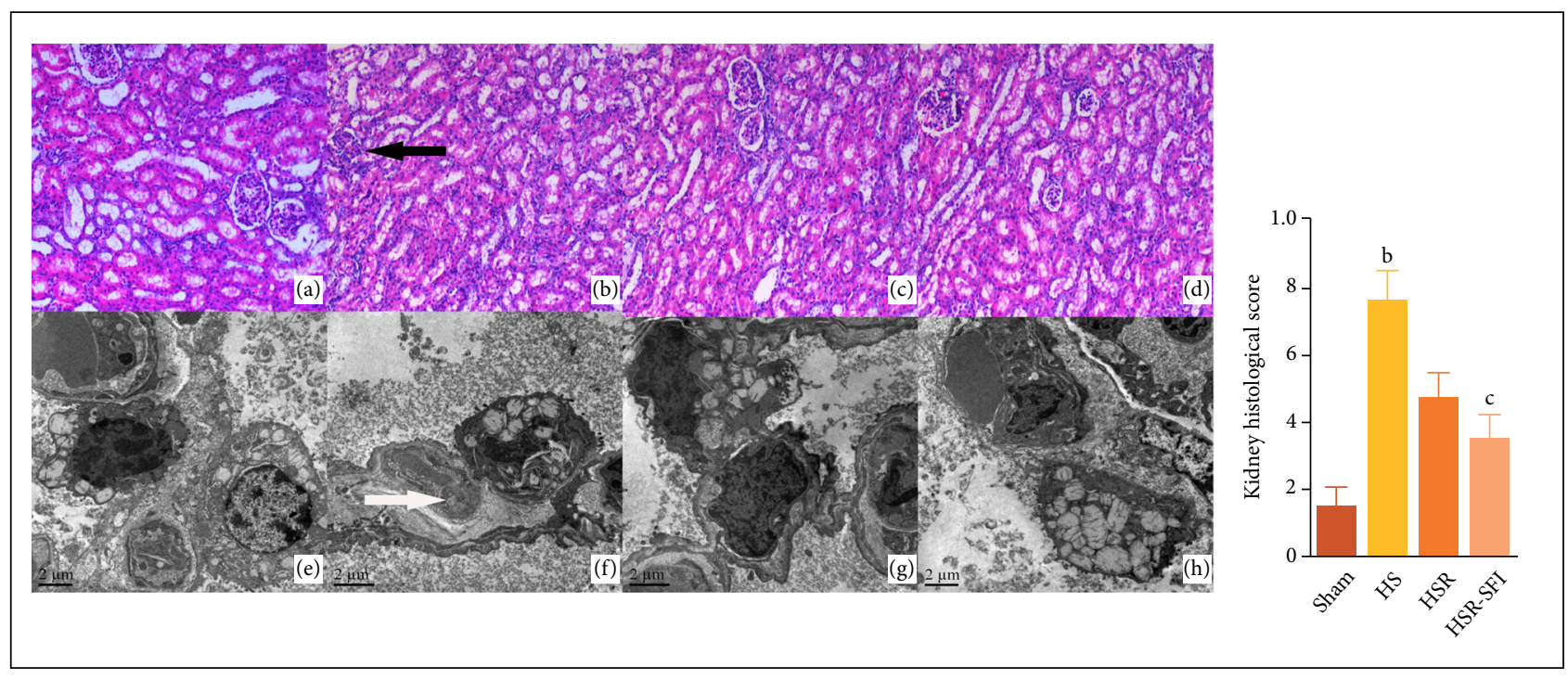

Figure 4 - The comparison of renal pathological results in four groups. HE staining and light microscopy findings: (a) Sham group; (b) HS group; (c) HSR group; (d) HSR-SFI group. Original magnification: 200x. Transmission electron microscopy results: (e) Sham group; (f) HS group; (g) HSR group; (h) HSR-SFI group. Original magnification: 2000x. In the HS groups, the results of light microscopy showed that the glomerular capillaries are closed, the whole glomerulus becomes smaller, and the endothelial cells of the glomerulus and the outer poetasters are in a state of pyknosis (black arrow). The renal microcystitis was widened, and there were some fine particles in the cavity. The epithelium of the proximal convoluted tubules is slightly lower than that of the normal ones; the intercellular boundary is not clear. A fine granular substance is scattered in the lumen. In the HS groups, the microvilli on the free surface of the proximal convoluted tubules were incomplete, the mitochondria in the epithelial cells were significantly expanded, the arrangement of mitochondria cristae was disordered, and even disappeared (white arrow). Owing to the expansion and deformation of mitochondria, the basal folds around them were disordered, and the lysosomes in the cells were increased. Autophagy was observed, and the deformed mitochondria were engulfed. $a, p<0.05$ vs. sham; $b, p<0.001$ vs. sham; $c, p<0.05$ vs. HSR; $d, p<0.001$ vs. HSR. Sham: the sham operation group, HS: the hemorrhagic shock group, HSR: the hemorrhagic shock and resuscitation group, HSR-SFI: the hemorrhagic shock and Shen-fu injection resuscitation group.

\section{Discussion}

This study confirmed that SFI reduces the risk of AKI due to hemorrhagic shock. Compared with the HSR group, the HSR-SF group produced more urine, had lower sCr levels, and lower plasma NGAL and CysC levels 4 and $6 \mathrm{~h}$ post shock, and their histopathologic scores were lower. Different from previous studies, according to these results, the protective effect of SFI on renal function not only comes from improving hemodynamics, but also may be related to attenuating systemic inflammatory response, and reducing apoptosis of renal tubular epithelial cells by regulating $\mathrm{Bcl}-2, \mathrm{BAX}$, and caspase-3 expressions.

According to previous studies, the AKI pathology during hemorrhagic shock and resuscitation is complex. In the early stage, AKl is mostly a function of trauma, hemorrhagic shock, and rhabdomyolysis. Later, as the injury progresses, it may relate more to a systemic inflammatory response, oxidative stress, and abdominal hypertension ${ }^{17}$.
In hemorrhagic shock, when the arterial pressure reaches the lower limit of autoregulation, renal blood flow decreases. Meanwhile, the increase of sympathetic activity and the release of renin and angiotensin lead to renal vasoconstriction, which worsens renal hypoxia ${ }^{18}$. No recognized optimal MAP exists for preserving renal function during resuscitation that follows hemorrhagic shock. However, when hemorrhaging is stopped, arterial pressure must be improved to optimize renal perfusion in case of vasoplegic shock ${ }^{17}$.

The results suggest that, compared with the standardtransfusion resuscitation, low-dose SFI and blood transfusion may increase MAP. Moreover, by comparing ITBVI between the two resuscitation groups, low-dose SFI did not increase the risk of pulmonary edema, which is a common complication in fluid resuscitation of hemorrhagic shock $^{19}$. This effect may relate to increased left ventricular ejection fraction and an improved hemodynamic index of the heart ${ }^{20,21}$. A higher MAP adequately perfuses the 
kidney and protects its function, which subsequently increases urine output.

Hemorrhage often precipitates systematic inflammatory response ${ }^{22}$, because it stimulates the immune system, which increases inflammation via secondary-messengers, changes in gene expression, and neutrophil activation ${ }^{23}$. The sustained and exacerbated inflammatory response may be deleterious to renal function following hemorrhagic injury. In these results, the cytokine levels of the HS group at 4 and $6 \mathrm{~h}$ post shock were significantly higher than those of the sham operation group. In the two resuscitation groups, cytokine levels were lower in the HSR-SF group. This difference suggests that SFI can reduce serum inflammatory mediator levels, including TNF- $\alpha$, IL-6, and IL-1 $\beta$ after HS and can attenuate excessive inflammatory responses.

The inflammasome-mediated, pro-inflammatory mediator releases contribute to the initiation, enhancement, and propagation of inflammation after trauma ${ }^{24}$, which promotes a further vascular endothelial barrier and parenchymal tissue damage ${ }^{25}$. This suggests that lower cytokine levels may reduce renal tissue damage post hemorrhage.

The relationship between apoptosis of the renal tubular epithelial cells and AKI is evident in studies on renal ischemia ${ }^{26,27}$. The mitochondrial pathway, as the "intrinsic" pathway of apoptosis ${ }^{28}$, controlled by the $\mathrm{Bcl}-2$ protein family, activates caspase- 3 when damaged mitochondria release cytochrome $\mathrm{c}$ in response to stress. BAX is a proapoptotic factor that participates in the mitochondrial apoptotic pathway. The Bcl-2, an antiapoptotic factor, can inhibit the function of $B A X^{29}$. The balance between $\mathrm{BCl}-2$ and $\mathrm{BAX}$ determines whether the intrinsic apoptosis pathway is initiated ${ }^{30}$.

The results show that SFI likely reduces the apoptosis of renal tubular epithelial cells by reducing the expression of $\mathrm{BAX}$, increasing the expression of $\mathrm{BCl}-2$, and inhibiting the expression of caspase-3. Similar findings have been reported in studies examining cardiac and lung tissues in a porcine model for cardiac arrest ${ }^{6,31}$ and in studies using a rat model to understand myocardial ischemia-reperfusion injury ${ }^{32}$.

\section{Limitations}

This study was focused on the functional protection of a single organ and did not reflect long-term outcomes. Consequently, it is not possible to infer that SFI can reduce the mortality of porcine HS model. This study did not compare outcomes to those of vasoactive drug interventions, such as norepinephrine or vasopressin, so it is not possible to determine if SFI exerts better protective effects on the kidney than other interventions. The animal experiments differ in setting, the injury was intentionally inflicted in a controlled environment, and the study setting differs from the clinical environment. Further research is needed to recommend a clinical protocol.

\section{Conclusion}

Shen-fu injection may reduce the risk of AKI following hemorrhagic shock by attenuating systemic inflammatory responses, and regulating the expression of apoptosisrelated proteins.

\section{Authors' contribution}

Design to the study: Yuan W, Wu JY, Zhang Q, and Li CS; Interpretation of data: Yuan W; Technical procedures: Yuan W, Wu JY, Zhang Q, and Li CS; Manuscript preparation: Li CS; Manuscript writing: Yuan W; Critical revision: Yuan W and Li CS; Final approval: Yuan W, Wu JY, Zhang Q, Liang $\mathrm{Y}$, Zhang MQ, Qin HJ and Li CS.

\section{Data availability statement}

Data will be available upon request.

\section{Funding}

Not applicable.

\section{Acknowledgments}

We would like to thank Editage (www.editage.cn) for English language editing.

\section{References}

1. Dutton RP, Stansbury LG, Leone S, Kramer E, Hess JR, Scalea TM. Trauma Mortality in Mature Trauma Systems: Are We Doing Better? An Analysis of Trauma Mortality Patterns, 1997-2008. J Trauma. 2010;69(3):620-6. https://doi.org/10.1097/TA.0b013e3181bbfe2a

2. Gomes E, Antunes R, Dias C, Araújo R, Costa-Pereira A. Acute kidney injury in severe trauma assessed by RIFLE criteria: a common feature without implications on mortality? Scand J Trauma Resusc Emerg Med. 2010;18:1. https://doi.org/10.1186/1757-7241-18-1

3. Rosenberger C, Goldfarb M, Shina A, Bachmann S, Frei U, Eckardt K-U, et al. Evidence for sustained renal hypoxia and transient hypoxia adaptation in experimental rhabdomyolysis-induced acute kidney injury. Nephrol Dial Transplant. 2008;23(4):1135-43. https://doi. org/10.1093/ndt/gfm808

4. van Meurs $M$, Wulfert FM, Jongman RM, Schipper $M$, Houwertjes MC, Vaneker M, et al. Hemorrhagic Shockinduced Endothelial Cell Activation in a Spontaneous 
Breathing and a Mechanical Ventilation Hemorrhagic Shock Model Is Induced by a Proinflammatory Response and Not by Hypoxia. Anesthesiology. 2011;115(3):47482. https://doi.org/10.1097/ALN.0b013e318229a640

5. Moreira MA, Irigoyen MC, Saad KR, Saad PF, Koike, MK, Montero EFS, et al. $\mathrm{N}$-acetylcysteine reduces the renal oxidative stress and apoptosis induced by hemorrhagic shock. J Surg Res. 2016;203(1):113-20. https://doi. org/10.1016/j.jss.2016.02.020

6. Zhang $M-Y$, Ji X-F, Wang S, Li C-S. Shen-Fu injection attenuates postresuscitation lung injury in a porcine model of cardiac arrest. Resuscitation. 2012;83(9):11528. https://doi.org/10.1016/j.resuscitation.2012.01.041

7. Thiyagarajan $\mathrm{P}, \mathrm{Chandrasekaran} \mathrm{CV}$, Deepak HB, Agarwal A. Modulation of lipopolysaccharide-induced pro-inflammatory mediators by an extract of Glycyrrhiza glabra and its phytoconstituents. Inflammopharmacol. 2011;19:235-41. https://doi.org/10.1007/s10787-011-0080-x

8. Li W, Li L, Chu Y-q, Al H-x, Ye C-f, Tong E, et al. Ginsenoside decreases expression of phosphorylated ERK and cytokine content in THP-1 cells induced by $\beta$-amyloid. Basic \& Clinical Medicine. 2006;26(4):362-5. https://doi. org/10.3969/j.issn.1001-6325.2006.04.005

9. Liu Z, Li Z, Liu X. Effect of ginsenoside Re on cardiomyocyte apoptosis and expression of $\mathrm{Bcl}-2 / \mathrm{Bax}$ gene after ischemia and reperfusion in rats. J Huazhong Univ Sci Technol [Med Sci]. 2002;22:305-9. https://doi.org/10.1007/ BF02896771

10. Ye J, Zhu Z, Liang Q, Yan X, Xi X, Zhang Z. Efficacy and safety of Shenfu injection for patients with return of spontaneous circulation after sudden cardiac arrest Protocol for a systematic review and meta-analysis. Medicine (Baltimore). 2018;97(38):e12500. https://doi. org/10.1097/MD.0000000000012500

11. Fan K-I, Wang J-h, Kong L, Zhang F-h, Hao H, Zhao H, et al. Effect of Shen-Fu Injection on Hemodynamics in Early Volume Resuscitation Treated Septic Shock Patients. Chin J Integr Med. 2019;25:59-63. https://doi.org/10.1007/ s11655-017-2965-z

12. Ling C, Li M, Tan J. Experimental study on protective effect of Chinese herbal medicine on glucocorticoid receptor. Chinese Journal of Integrated Traditional and Western Medicine. 1999;19(5):302-3.

13. World Medical Association. World Medical Association Declaration of Helsinki: Ethical Principles for Medical Research Involving Human Subjects. JAMA. 2013;310(20):2191-4. https://doi.org/10.1001/ jama.2013.281053

14. Roesner JP, Petzelbauer P, Koch A, Tran N, Iber T, Vagts DA, et al. Bß15-42 (FX06) reduces pulmonary, myocardial, liver, and small intestine damage in a pig model of hemorrhagic shock and reperfusion. Crit Care Med. 2009;37(2):598605. https://doi.org/10.1097/CCM.0b013e3181959a12
15. Wu J, Li C, Yuan W. Effects of Shenfu injection on macrocirculation and microcirculation during cardiopulmonary resuscitation. J Ethnopharmacol. 2016;180:97-103. https:// doi.org/10.1016/j.jep.2016.01.027

16. Parlakpinar $H$, Acet $A$, Gul $M$, Altinoz E, Esrefoglu $M$, Colak C. Protective effects of melatonin on renal failure in pinealectomized rats. Int J Urol. 2007;14(8):743-8. https://doi.org/10.1111/j.1442-2042.2007.01806.x

17. Harrois A, Libert N, Duranteau J. Acute kidney injury in trauma patients. Curr Opin Crit Care. 2017;23(6):447-56. https://doi.org/10.1097/MCC.0000000000000463

18. Hultström M. Neurohormonal interactions on the renal oxygen delivery and consumption in haemorrhagic shock-induced acute kidney injury. Acta Physiol (Oxf). 2013;209(1):11-25. https://doi.org/10.1111/apha.12147

19. Santry HP, Alam HB. Fluid Resuscitation: Past, Present, and the Future. Shock. 2010;33(3):229-41. https://doi. org/10.1097/SHK.0b013e3181c30f0c

20. Yan X, Wu H, Ren J, Liu Y, Wang S, Yang J, et al. Shenfu Formula reduces cardiomyocyte apoptosis in heart failure rats by regulating microRNAs. J Ethnopharmacol. 2018;227:10512. https://doi.org/10.1016/j.jep.2018.05.006

21. Zhang Q, Li C. The Roles of Traditional Chinese Medicine: Shen-Fu Injection on the Postresuscitation Care Bundle. Evid Based Complement Alternat Med. 2013;2013:319092. https://doi.org/10.1155/2013/319092

22. Namas R, Ghuma A, Hermus L, Zamora R, Okonkwo DO, Billiar TR, et al. The Acute Inflammatory Response in Trauma / Hemorrhage and Traumatic Brain Injury: Current State and Emerging Prospects. Libyan J Med. 2009;4:13648. https://doi.org/10.4176/090325

23. Baroja-Mazo A, Martín-Sánchez F, Gomez Al, Martínez CM, Amores-Iniesta J, Compan V, et al. The NLRP3 inflammasome is released as a particulate danger signal that amplifies the inflammatory response. Nat Immunol. 2014;15:738-48. https://doi.org/10.1038/ni.2919

24. Kruger $P$, Saffarzadeh $M$, Weber ANR, Rieber N, Radsak M, von Bernuth $\mathrm{H}$, et al. Neutrophils: Between Host Defence, Immune Modulation, and Tissue Injury. PLoS Pathog. 2015;11(3):e1004651. https://doi.org/10.1371/journal. ppat. 1004651

25. Sato H, Tanaka T, Kasai K, Kita T, Tanaka N. Role of p38 Mitogen-Activated Protein Kinase on Renal Dysfunction After Hemorrhagic Shock in Rats. Shock. 2005;24(5):48894. https://doi.org/10.1097/01.shk.0000176594.64626.7f

26. Zhou Y, Cai T, Xu J, Jiang L, Wu J, Sun Q, et al. UCP2 attenuates apoptosis of tubular epithelial cells in renal ischemiareperfusion injury. Am J Physiol Renal Physiol.2017;313:F92637. https://doi.org/10.1152/ajprenal.00118.2017

27. Qin C, Xiao C, Su Y, Zheng H, Xu T, Lu J, et al. Tisp40 deficiency attenuates renal ischemia reperfusion injury induced apoptosis of tubular epithelial cells. Exp Cell 
Res. 2017;359(1):138-44. https://doi.org/10.1016/j. yexcr.2017.07.038

28. Sawant DA, Tharakan B, Hunter FA, Childs EW. The Role of Intrinsic Apoptotic Signaling in Hemorrhagic Shock-Induced Microvascular Endothelial Cell Barrier Dysfunction. J Cardiovasc Transl Res. 2014;7:711-8. https://doi.org/10.1007/s12265-014-9589-x

29. Dillon CP, Green DR. Molecular Cell Biology of Apoptosis and Necroptosis in Cancer. In: Gregory C, editor. Apoptosis in Cancer Pathogenesis and Anti-cancer Therapy. Advances in Experimental Medicine and Biology. Cham: Springer; 2016. p. 1-23. https://doi.org/10.1007/978-3319-39406-0_1
30. Llambi F, Green DR. Apoptosis and oncogenesis: give and take in the BCL-2 family. Curr Opin Genet Dev. 2011;21(1):1220. https://doi.org/10.1016/j.gde.2010.12.001

31. Wu Y, Xia Z-y, Meng Q-t, Zhu J, Lei S, Xu J, et al. Shen$\mathrm{Fu}$ Injection Preconditioning Inhibits Myocardial Ischemia-Reperfusion Injury in Diabetic Rats: Activation of eNOS via the PI3K/Akt Pathway. J Biomed Biotechnol. 2011;2011:384627. https://doi. org/10.1155/2011/384627

32. Wang Y-I, Wang C-y, Zhang B-j, Zhang Z-z. Shenfu injection suppresses apoptosis by regulation of $\mathrm{Bcl}-2$ and caspase-3 during hypoxia/reoxygenation in neonatal rat cardiomyocytes in vitro. Mol Biol Rep. 2009;36:365-70. https://doi.org/10.1007/s11033-007-9188-x 